\title{
Capacity improvement of the single mode air interface WCDMA FDD with relaying
}

\author{
H. Nourizadeh, S. Nourizadeh and R. Tafazolli \\ Centre for Communication Systems Research (CCSR) \\ University of Surrey, Guildford, UK \\ E-mail: h.nourizadeh@eim.surrey.ac.uk
}

\begin{abstract}
A new way to model a CDMA-system applying relaying is proposed in this paper. This method makes it possible to compare directly the performance of relaying. The outage probability, which represents the ability of the users to reach the base station, is chosen as criteria to compare the system with and without relaying. The model is based on the single mode air interface WCDMA FDD with a two-hop relay. When relaying is applied, the simulation results show that even by using the single mode FDD the uplink capacity is significantly improved by $82 \%$. Also a new relay node selection strategy is proposed and the results show how important it is to choose appropriately the relay node. And finally, different scenarios of relaying are simulated to show when or when it is not better to apply relaying.
\end{abstract}

\section{INTRODUCTION}

In a CDMA system, all users interfere with each others. Therefore the CDMA system is interference limited. The users, which suffer from a strong shadow effect or because they are far away from the BS, need more power to reach the latter. In this case, theses users may transmit at their maximum allowed power without satisfying their Quality of Service. Therefore, this would leave some mobiles out of the system and also create too much interference to the neighboring cells.

One-way to counter this problem and therefore to improve the capacity would be to increase the numbers of BS. But this solution cannot be efficient as it increases significantly the network infrastructure cost. Another way to improve the capacity is a system applying relaying [1]. This means using other existing terminals located between the originating terminal and the BS for the purpose of retransmitting the original packet. With this process, the users at the boundaries of the cells will need less power to reach the relay station than to reach the BS and therefore create less interference to the neighboring cells, hence improving the capacity.

In the last few years, there has been a great interest in cellular networks applying relaying. First, ODMA (Opportunity Driven Multiple Access), which is an intelligent protocol that sits upon a radio sub-system that support relaying, was proposed. One node relays its packets through others nodes by using ODMA with a separate unpaired spectrum band and the node the closer to the BS sends the packet by using the TD/CDMA-FDD mode [1]. The Ad Hoc GSM cellular system adds the relay capability to a second generation GSM network to enhance the system coverage [2]. The iCAR system can efficiently balance traffic loads between cells by using fixed relay station to relay traffic [3].

The system proposed in this paper is based on a two-hop relay cellular network. In this work, the relay stations are the mobile station non-communicating with the BS. The positive point of using MS as relay is with a great number of users in the system, a MS has more chance to choose an appropriate MS relay. With fixed relay station, the users far away may have difficulties to reach the fixed relay station. If a MS relays its packet through another MS, it can considerably decrease the battery of the MS relay. One solution to this problem would be to employ cars or taxi as relay station, since they have infinite battery. In this system, the air interface WCDMA FDD is used for the two hops. With the current technology this is not applicable, because wireless terminals cannot transmit and receive in the same frequency band in the mode FDD. However some changes can be made at the hardware of the mobile, like using two-duplexer, in order to apply it in the single mode FDD. Also it can be interesting to see if the capacity is improved even by using a single mode, which is the worse case, because each hop interferes with each other. Section II describes the system model proposed. Section III explains the different schemes to model relaying. The results are shown in section IV. And finally, a conclusion is given in section $\mathrm{V}$.

\section{MulTi-HOP BASED CELIULAR NeTWORKS}

\section{A. System description}

The Figure 1 (a) and (b) depict the scenario of the simulation model. The Figure 1(a) shows the MS active and non-active in a single cell without relaying. The MS active are sending continuously data information to the BS. With relaying, different MS role are identified such as: 
- MO (Mobile Originator/Outage), which have a weak link with the BS.

- $M I$ (Mobile Intermediate), which can reach the BS.

- MS non-active, which are mobile not sending data to the BS.

With the same location for all the users, the MOs look for a MS non-active to relay their information, see Fig. 1 (b). In this model, one MS relay can relay only one MO. With this scenario, at each instant the performance of the system with and without relaying can directly be compared for the same position for all users.

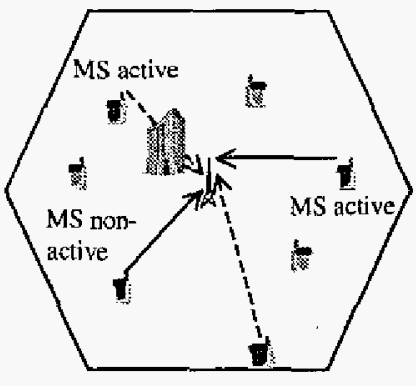

(a) Without relaying

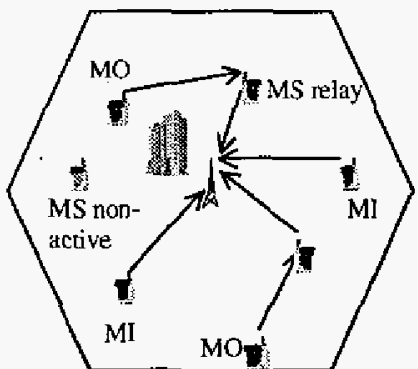

(b) With relaying
Fig. 1. Simulation model

\section{B. System model}

A MS is in outage if its SIR (Signal-to-Interference ratio) is below a certain threshold. At any time, the SIR of all

MS active can be calculated. If $G$ denote the gain, $P$ denote the transmitter power and the uplink measured intracell and intercell interference at the BS $i$ are denoted by $I_{\text {intracell }}^{i}$ and $I_{\text {intercell }}^{\mathrm{i}}$ respectively, the average $\mathrm{SIR}$ of user $u$ is:

$$
S I R_{\text {iu }}=\frac{G_{i u} P_{i u}}{I_{\mathrm{int} \text { racell }}^{i}+I_{\mathrm{int} \mathrm{ercell}}^{i}+n_{s}}
$$

where $I_{\text {itr racell }}^{i}=\sum_{j \neq i}^{N^{j}} G_{i j} P_{i j}$, with $N^{i}$ represents the number of users that belong to the BS $i$; and $I_{\text {intercell }}^{i}=\sum_{m \neq i}^{M} \sum_{k=1}^{N^{m}} G_{i k} P_{i k}$, with $N^{m}$ the number of users that belong to the BS $m$, and $M$ the number of cell in the system. $n_{s}$ represents the background noise.

If the received SIR of a MS is below its SIR threshold, this MS is considered in outage. This means that the signal of the MS is not strong enough to overcome the interference received by the $\mathrm{BS}$.

In order to compare the two systems, with and without relaying, the SIR of the links MI-BS, MO-MS relay and MS
relay-BS need to be calculated. As the MIs and the MSs relay send their signal to the BS they have the same interferers, and their SIR can be expressed as:

$$
S I R_{i u}=\frac{G_{i u} P_{i u}}{I_{M I}^{i}+I_{M S_{\text {retoy }}}^{i}+I_{M O}^{i}+I_{\text {intercell }}^{i}+n_{s}}
$$

where $I_{M \prime}^{i}, I_{M S_{\text {play }}}^{i}$ and $I_{M O}^{i}$ represent the intracell interference at the BS $i$ respectively from the MI, MS relay and MO from the same cell.

If the MO $u$ sends its signal to the MS relay $r$ within the cell of the BS $i$, its SIR can be expressed as:

$$
S I R_{r, \omega}^{i}=\frac{G_{r u}^{i} P_{r u}^{i}}{I_{r, M I}^{i}+I_{r, M S_{r e l o y}}^{i}+I_{r, M O}^{i}+I_{r, \text { int ercell }}^{i}+n_{s}}
$$

where $I_{r, M i}^{i}, I_{r, M S_{\text {rloy }}}^{i}$ and $I_{r, M O}^{i}$ represent the intracell interference at the MS relay $r$ respectively from the MI, MS relay and MO in the cell of the BS $i$.

\section{MULTI-HOP RELAYING SCHEMES}

In this section, three different issues on a two-hop cellular network are discussed. In the part A, improving the link between the terminals by applying power control is presented. The MS relay selection, based on the SIR and the distance, is discussed in the part B. Finally in part C, possible methods to reduce the number of relayed MS are discussed.

\section{A. Power control between $M S$ and $M S$ relay}

Until nowadays, few have considered in the literature to adapt the transmit power between two MSs, communicating with each other. Here, it is considered the case where MO is capable of adjusting its transmit power according to its received SIR at the MS relay. A perfect distributed power control has been modeled for the links between MS and BS and also between MS and MS relay. The power control objective between MS and MS relay is similar than traditional power control algorithms used in CDMA-based cellular systems. It consists of balancing all the received SIR around the SIR target value, [4]. Power control is performed in subsequent iterations and the transmitter power $P_{u}^{(n+1)}$ of each user $u$ is adjusted at iteration $n+I$ according to

$$
P_{u}^{(n+1)}=\frac{\Gamma}{\gamma_{u}^{(n)}} P_{u}^{(n)}
$$

where $\gamma_{u}^{(n)}$ is the measured SIR of the link at iteration $n$ and $\Gamma$ is the target SIR for the link. 


\section{B. MS relay selection strategy}

The choice of the MS relay is an important issue in multi-hop cellular networks. In [5] the criteria to determine the routing is based on minimizing interference. In this paper, a new algorithm based on the maximum SIR overall and on the distance between users is introduced. Within a certain range, each MO will choose the MS non-active with the maximum SIR overall as a MS relay.

\section{C.1. Relay $M S$ in the overlap area}

The figure 2 shows the CDF (Cumulative Distribution Function) of the distance between the MO and the BS. The curve shows that most of the MOs are far away from the BS. $70 \%$ of the MOs are distant from above $1600 \mathrm{~m}$. The MO cannot counter the loss along the path and reach the BS even with the maximum power. Not only the signal received at the BS is weak but also they create a lot of intercell interference by using their maximum power.

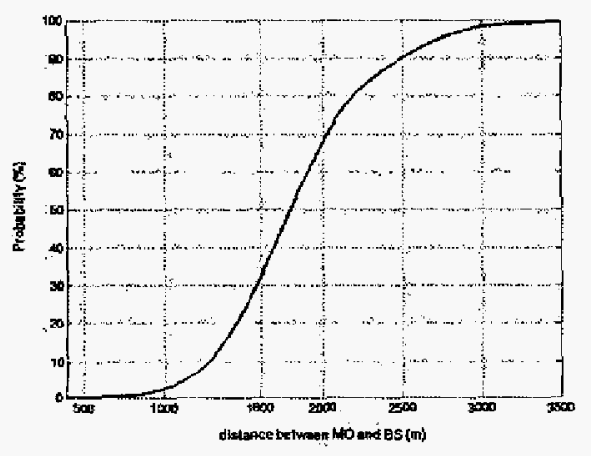

Fig. 2. CDF of distance between $\mathrm{MO}$ and $\mathrm{BS}(\mathrm{m})$

\section{a) Only $\mathrm{MO}$}

$B y$ relaying only the MOs far away from the $B S$, not only the intercell interference will decrease but also the SIR of MO not in the overlap area will increase and some of them may not be in outage without the need of relaying (see Fig. 3).

b) All MS active

There are some MS active in the overlap area, which are not in outage, but because of a high propagation loss, they transmit with a great power. Therefore, they create also a lot of interference to the neighboring cells. These MS active, even if they are not in outage, can be relayed in order to use less transmit power and therefore decreasing the intercell interference. A new scheme is proposed where all the MS active (in outage and not in outage) in the overlap area are relayed.

\section{C.2. Relay $M S$ with SIR $<S I R_{-}$ref}

A final scheme analyzed in this paper, proposes to relay only the MOs, which have the lowest received SIR. For this, a new threshold SIR_reference is defined that is lower than the SIR target. The idea is to relay only the MOs with a SIR below the SIR_reference. With this process, the SIR of the MOs not relayed can be increased and theses MO can achieve the target when relaying is done for only the MOs with a SIR below the SIR_reference.

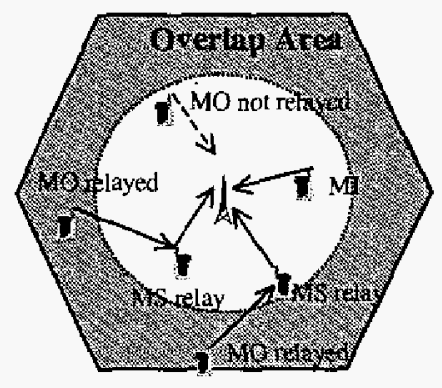

Fig. 3. Relaying only MO in the overlap area

\section{Simulation Results}

\section{A. Simulation Environment}

Cell planning is an important issue when relaying needs to be implemented in cellular networks. The overlap area between neighboring cells should be kept to the minimum so that the system experiences minimum interference. Therefore a link budget for the vehicular environment, as in [6], is performed in order to find the most accurate value for the radius of the cell.

The system modeled includes 19 hexagonal cells of radius $2000 \mathrm{~m}$, with BS at the center of the cells employing omni directional antenna. The MS follow the mobility and the propagation model as proposed in [7] for the vehicular environment and for the hard handover case. A new auto correlated shadowing model in 2 dimensions is modeled between the MS and the BS, as in [8]. There are no appropriate path loss models that describe the channel between MS since a mobile terminal is usually positioned around head. However, the Lee model, which takes account the receivers antenna height, is chosen as model [9].

The model is simulated at system level and the simulation has been run for 10000 iterations. The simulation result is based on outage probability, which represents the number of MS in outage over the total number of MS active for each cell. At the end of the simulation, the outage probability calculated after each time with and without relaying for the seven central cells are averaged and compared.

\section{B. Results}

I) PC between MS-MS relay

Figure 4 shows the outage probability with and without relaying for different number of active users in each cell.

The results show that the outage probability decreases when relaying is applied. For instance, at $10 \%$ of outage probability, the number of active users supported in the cell without relaying is 28 but with relaying (No $\mathrm{PC}$ ) it is 32 , which shows an improvement of $14 \%$. In this case, the MO 
transmit to the MS relay with the same fixed power, which is the maximum power allowed. Relaying with power control between MS shows a significant improvement of $32 \%$. For $10 \%$ outage probability, the number of users active with relaying and with power control supported is 37 . With power control between the MS, the MO will transmit with the minimum power needed to reach its MS relay.

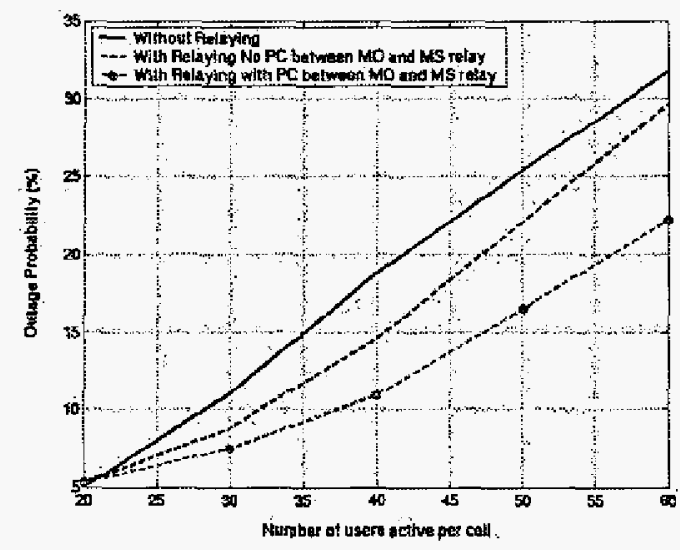

Fig. 4, Outage probability vs. Number of users active per cell with power control between MSs

2) Interference study

Simulations have been performed to study the intracell and intercell interference with and without relaying. The interference ratio (intercell/intracell interference) calculated without relaying is approximately 1.7 , which is in agreement with $[10]$ for the hard handover case. The intracell interference is increased from $-117.2 \mathrm{~dB}$ to $-116.6 \mathrm{~dB}$ when relaying is applied. With relaying, the MS relay, which relays the $\mathrm{MO}$, can achieve the target and then send a stronger signal to the BS than the MO without relaying, which increase the intracell interference. On the other hand, since smaller distances need to be covered when relaying is applied, the intercell interference is decreased from $-121.1 \mathrm{~dB}$ to -123 $\mathrm{dB}$.

\section{3) MS relay selection schemes}

A new selection scheme based on highest SIR overall and the distance between the MO and the MS relay has been tested. In this algorithm, the MO finds a MS relay within a certain range and selects the one with the highest SIR for the twohop relay. Figure 5 shows the outage probability without relaying, with relaying based on the highest SIR overall without range and with different allowed ranges (maximum distance) of $500 \mathrm{~m}, 1000 \mathrm{~m}$ and $1500 \mathrm{~m}$. This figure shows a significant improvement when the relay selection scheme is based on the maximum SIR and distance. When the maximum distance to find a MS relay is set to $1500 \mathrm{~m}$, the MO can easily find a MS relay in this range but the distance is stitl too large in order for the system to operate efficiently. With the maximum distance set to $500 \mathrm{~m}$, the entire MO may not be able to find a MS relay in that range and will select a MS relay far away which will lead to high transmission power. A good compromise has been found to be approximately $1000 \mathrm{~m}$. All $\mathrm{MO}$ will be able to find a MS relay in this range, which is half of the cell radius. For $10 \%$ outage probability, the number of active users supported in a cell with the relay selection scheme based on the overall highest SIR and the distance between the MO and the MS relay is 51 (with maximum distance $=1000 \mathrm{~m}$ ), which leads to a significant improvement of $82 \%$.

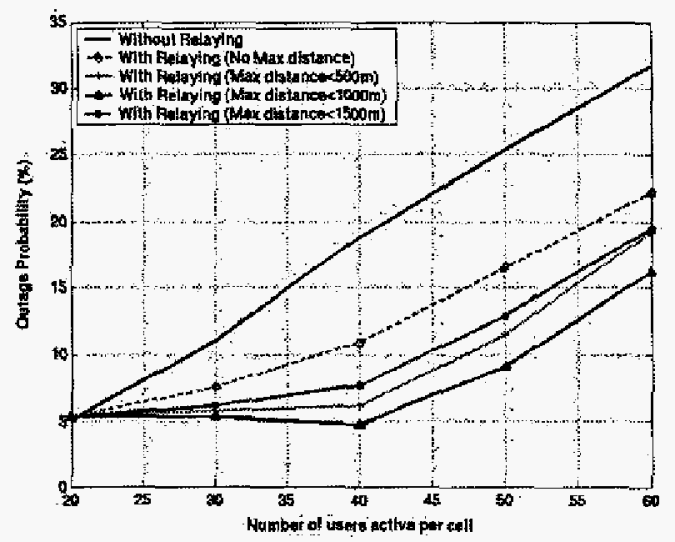

Fig. 5. Outage probability vs. Number of users active per cell with different range to find a MS relay

4) Relaying only $M O$ in the overlap area

Figure 6 shows the outage probability in terms of the number of active users without relaying, with relaying all MS active in the overlap area, with relaying only $\mathrm{MO}$ that are in the overlap area, with relaying only MO with SIR $<$ SIR_reference and with relaying all $\mathrm{MO}$.

The results show that the performance is better when relaying is performed for all the MO, and not only the MO in the overlap area. For instance for $10 \%$ outage probability, the numbers of active users in a cell with relaying only $\mathrm{MO}$ in the overlap area is 33, but with relaying all MO it's 51 . There are some MO close to the BS that achieve communication without relaying because the intercell interference is decreased but not all of them since the intracell interference is increased with relaying.

The radius of the circle that defines the overlap area is an important factor. The bigger the circle is, the less relaying will be performed and the outage probability with relaying only $\mathrm{MO}$ in the overlap area will be equal to the outage probability without relaying. But the smaller is the circle, the more relaying will be performed and the outage probability with only $\mathrm{MO}$ relaying in the overlap area will be equal to the outage probability with relaying.

Even if the performance is better with relaying for all $\mathrm{MO}$, it may be interesting to model these schemes in a system where the signaling overhead is included in the simulation. Since the overhead will increase with relaying, the capacity of 
a system may not improve if the overhead is too high, as shown in [11]. Therefore, the system designer can select only some MO to relay.

\section{5) Relaying all MS active in the overlap}

Figure 6 shows that the outage probability when relaying all active MS is higher than the outage probability when relaying all the MOs. The performance is worse because some active MS which are not in outage may use a higher overall transmit power with relaying than a direct link without relaying. Also one of the MS active-MS relay or MS relay-BS links can fail which leads to this active MS to go into outage whereas it was not in outage with a direct link.

\section{6) Relaying only $M O$ with $S I R<S I R_{-} r e f$}

The results in Fig. 6. show that the best performance is obtained when all the MO are relayed. It is interesting to observe that the outage probability when relaying is applied for the MO with SIR below the reference SIR is less than when relaying is applied to the $\mathrm{MO}$ in the overlap area. Some MO that are close to the BS but exhibit very low SIR cannot achieve the target. But relaying MO with a small SIR can increase the SIR of the MOs not relayed with a SIR close to the target. This means the Signal-to-Interference is a better criterion than distance.

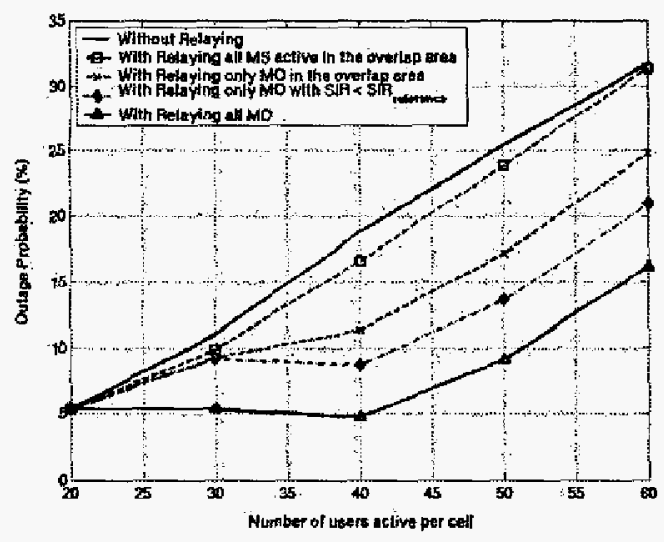

Fig. 6. Different schemes to relay only some MO. (The MS relay selection strategy is based on the highest SIR and the distance maximum equal to $1000 \mathrm{~m}$ )

Figure 7 shows the SIR of the MOs not relayed without relaying and with relaying only the MO with a SIR $<$ SIR_ref. The SIR target is equal to $-21 \mathrm{~dB}$ whereas the SIR reference is set at $-23 \mathrm{~dB}$. The MOs not relayed have a SIR between $-23 \mathrm{~dB}$ and the SIR target $-21 \mathrm{~dB}$. But with relaying all $\mathrm{MO}$ with a SIR below the SIR reference $(-23 \mathrm{~dB})$, the MO not relayed increase their SIR since the interference decreases. The curve with relaying shows that $60 \%$ of the MO not relayed has a SIR above the target $(-21 \mathrm{~dB})$. This shows that most of the MOs establish a link with the BS without the need of relaying.

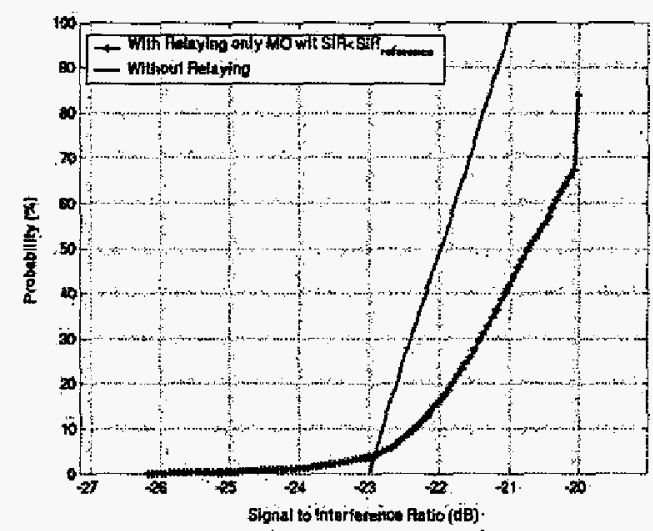

Fig. 7. CDF of the MOs not relayed.

\section{CONCLUSION}

The results showed that relaying could significantly improve the capacity even by using the single mode FDD for the twohop relay. A new scheme to choose the most appropriate relay has been proposed. This latter emphasized the importance of the relay node selection strategy. Different aspects of relaying integrated to cellular systems were discussed and their advantages demonstrated. It was shown that a combination of these proposed techniques such as applying power control between two MS, a routing selection based on both SIR and distance and finally relaying all the MOs can provide a capacity gain up to $82 \%$.

\section{ACKNOWLEDGMENT}

The authors gratefully acknowledge the support of Vodafone (UK) to make possible to do this research.

\section{REFERENCES}

[1] ETSI.TR 101146 v3.0.0 "UMTS 30.06, Support for relaying and ODMA" December 1997.

[2] G. Aggelou and R. Tafazolli, "On the relaying capability of next generation GSM cellular nctworks" IEEE Personal Communications, vol. $8, \mathrm{pp} 40-47,2001$

[3] H. Wu, C. Qias, S. De, O. Tonguz "iCAR; Integrated Cellular and Ad hoc Relaying systems" IEEE joumal on selected areas in communications, October 2001 .

[4] J. Zander, "Distributed cochannel interference control in cellular radio systems" JEEE Trans. Veh. Technol., vol. 41, pp. 57-62,1992.

[5] T. Rouse, I. Band, S. McLaughlin "Capacity and power investigation of ODMA in UTRA TDD" IEE 3G 2001, pp 252-256.

[6] J. Laiho, A. Wacker, and T. Novosad, Radio Network planning Optimisation for UMTS. Wiley, 2002. p82.

[7] ETSI (UMTS); selection pmocedures for the choice of nadio transmission technology of the UMTS (UMTS 30.03 v3.2.0) 1998-04.

[8] H. Nourizadeh, X. Yang and $\mathbf{R}$. Tafazolli " Generation of two-dimensional shadowing for dynamic system-level simulators" submitted for IEEE Communications fetters.

[9] S.C Yang "CDMA RF Engineering", Artech House, Boston London, 1998.

[10] A. Viterbi, A. Viterbi, K. Gilhousen, and E. Zehavi, "Soft Handoff extends CDMA cell coverage and increases reverse link capacity", IEEE Joumal on Sel. Areas in Cormm., vol, 12, pp 1281-1288, October 1994.

[11] O. B. Holm, T. Friiso, and T. Haslestad, "Improving UTRA Capacity with ODMA", IST Mobile Communications Summit, 2002. 